\title{
Developmental Venous Anomaly With Asymmetrical Basal Ganglia Calcification: Two Case Reports and Review of the Literature
}

\author{
Ali Firat Sarp ${ }^{1, *} ;$ Ozan Batki $^{1}$; Mustafa Fazil Gelal ${ }^{1}$ \\ ${ }^{1}$ Department of Radiology, Ataturk Training and Research Hospital, Izmir Katip Celebi University, Izmir, Turkey \\ ${ }^{*}$ Corresponding author: Ali Firat Sarp, Department of Radiology, Ataturk Training and Research Hospital, Izmir Katip Celebi University, Izmir, Turkey. Tel: +90-5064417980, \\ E-mail: firat_sarp@yahoo.com
}

Received: December 15, 2013; Revised: March 27, 2014; Accepted: May 28, 2014

\begin{abstract}
Developmental venous anomaly (DVA) is a common lesion formerly known as venous angioma. DVAs drain normal brain parenchyma; however, parenchymal abnormalities surrounding DVAs have been reported. Unilateral putamen and caudate calcification in the drainage territory of DVAs has so far been reported in 7 cases, all with deep venous drainage. We present two additional cases of DVAs, one with superficial and the other one with deep venous drainage, associated with basal ganglia calcifications. We emphasize that DVAs should be in the differential diagnosis of unilateral basal ganglia calcifications.
\end{abstract}

Keywords: Central Nervous System Venous Angioma; Magnetic Resonance Imaging; Vascular Malformations; Idiopathic Basal Ganglia Calcification

\section{Introduction}

Developmental Venous Anomaly (DVA) is a common lesion formerly known as venous angioma (1-5). DVAs drain normal brain parenchyma; however, parenchymal abnormalities surrounding DVAs have been reported $(1,3$, 6). Unilateral putamen and caudate calcification in the drainage territory of DVAs has so far been reported in 7 cases, all with deep venous drainage $(1,3)$. We present a unique case of unilateral putamen and caudate calcification associated with a superficially drained DVA and an additional case with asymmetrical lentiform nucleus calcification accompanied by a deep drained DVA and show that these calcification patterns can be seen in the presence of DVAs, regardless of the drainage site. We also emphasize that DVAs should be in the differential diagnosis of unilateral basal ganglia calcifications.

\section{Case Presentation}

\subsection{Case 1}

An 85-year-old male patient, with longstanding tensiontype headache, underwent contrast-enhanced magnetic resonance imaging (MRI) of the brain. The MRI scan showed generalized cerebral atrophy and an incidental DVA in the left putamen. The lesion consisted of radially oriented contrast enhancing small vessels draining the left basal ganglia, which terminated in a larger collector vein. A focal narrowing was noted at the site where the large collector vein drained into superficial middle cerebral vein (Figure $1 \mathrm{~A}$ ). Axial gradient-recalled echo (GRE) T2-weighted images showed bilateral symmetrical low signal at globus pallidi and low signal at left-sided putamen and caudate (Figure $1 \mathrm{~B}$ ). Head computed tomography (CT) confirmed that low signal seen at GRE T2 corresponded to calcifications (Figure $1 \mathrm{C}$ ). MRI and CT were otherwise normal. There was no abnormality in routine biochemical analysis and serum calcium level was normal.

\subsection{Case 2}

An 81-year-old man with dementia was referred to our hospital for the diagnostic work-up of the left-sided basal ganglia hyperdensity seen at head CT, which was interpreted as being suspicious for hematoma. He did not have any neurologic abnormalities. MRI showed brain atrophy and chronic ischemic white matter changes. Maximum intensity projection (MIP) of the contrast enhanced-MR venography showed a DVA in the territory of left lentiform nucleus, draining into left internal cerebral vein (Figure 2 A). Susceptibility weighted imaging (SWI) showed low signal in bilateral lentiform nuclei, which was dominant on the left side (Figure $2 \mathrm{~B}$ ). CT proved that areas with low signal on SWI were calcifications (Figure 2 C). There was no evidence of a hematoma. Serum calcium and phosphate levels were within normal limits.

Copyright (C) 2015, Tehran University of Medical Sciences and Iranian Society of Radiology. This is an open-access article distributed under the terms of the Creative Commons Attribution-NonCommercial 4.0 International License (http://creativecommons.org/licenses/by-nc/4.0/) which permits copy and redistribute the material just in noncommercial usages, provided the original work is properly cited. 

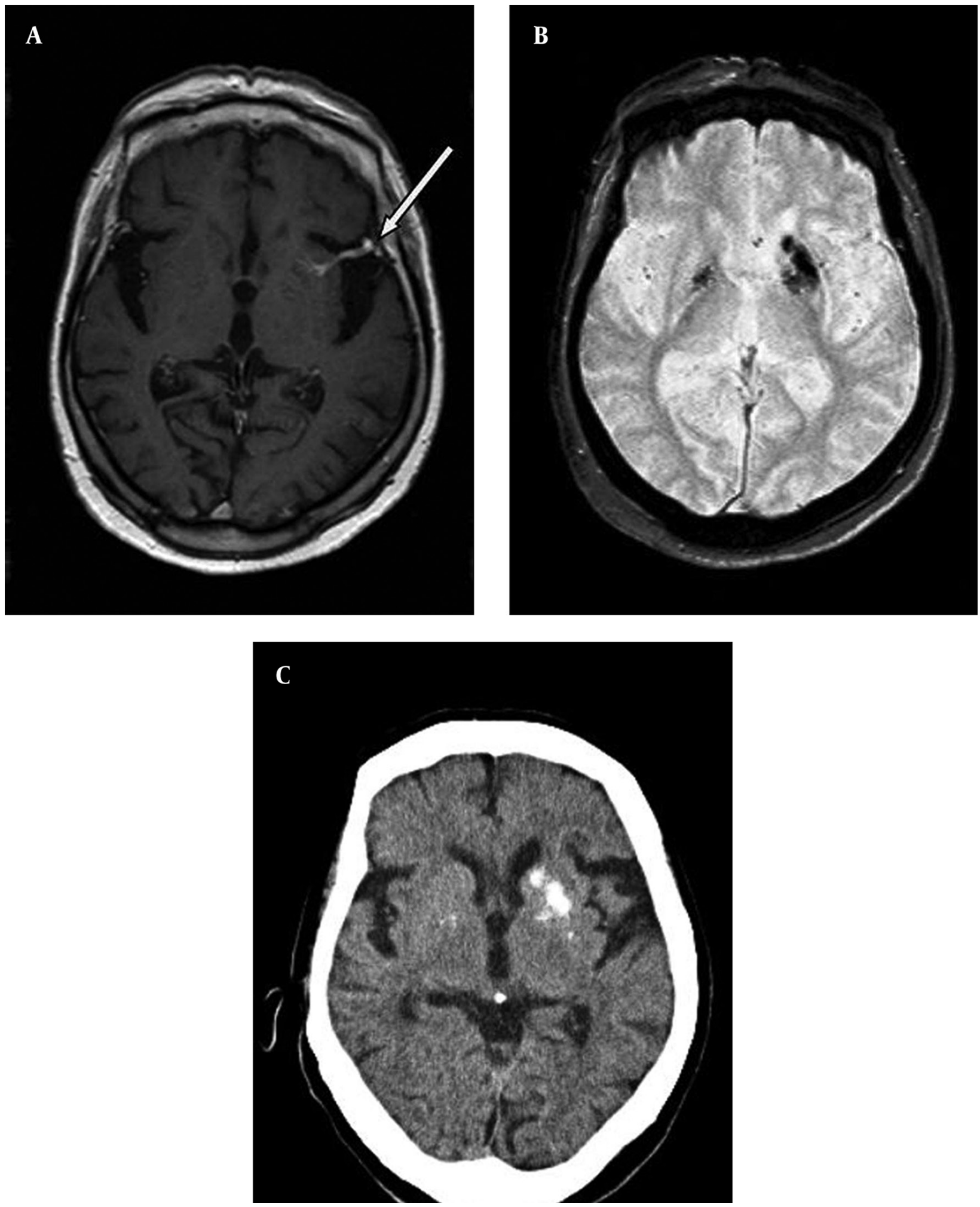

Figure 1. An 85-year-old male patient with longstanding tension headache, underwent brain MRI. A, postcontrast axial T1-weighted MRI reveals a DVA in left putamen with focal narrowing of large collector vein at drainage site into superficial vein (arrow). B, axial gradient-recalled echo T2 shows low signal at bilateral globus pallidi and unilateral left putamen and caudate. C, brain CT shows that low signal seen at axial gradient-recalled echo T2 corresponds to calcification. 

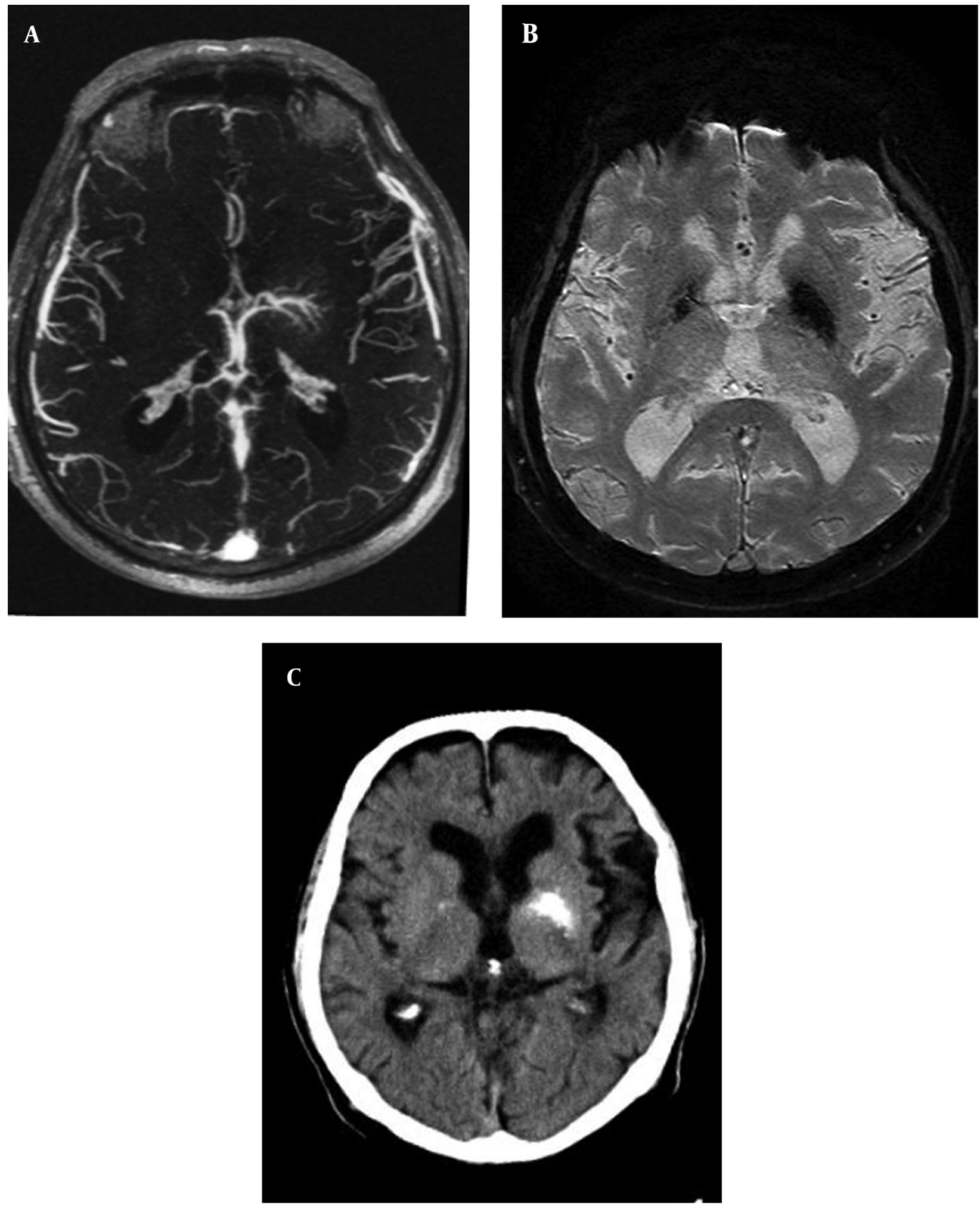

Figure 2. An 81-year-old man with dementia and basal ganglia hyperdensity in CT suspicious for hematoma. A, maximum intensity projection-intensity projection magnetic resonance venography shows a DVA in the left lentiform nucleus, collector vein of the developmental venous anomaly drains into left internal cerebral vein. B, susceptibility-weighted imaging sequence reveals bilateral lentiform nucleus apparent signal loss, significantly dominant on the left side. C, brain computed tomography demonstrates that low susceptibility-weighted imaging signals correspond to calcification. 


\section{Discussion}

DVA is the most common vascular malformation of the brain occurring in up to $4 \%$ of general population and is usually discovered incidentally by contrast-enhanced MRI $(1,4,7)$. Some authors consider DVAs as embryological variants of venous drainage system $(4,5,7)$. They are characterized by radially oriented dilated veins draining normal parenchyma, which are typically likened to "caput medusa" and a larger collector vein, which drains into superficial or deep venous system. Although DVAs are usually asymptomatic, headache, seizures and focal neurological deficits have been claimed to be associated with DVAs (4).

Some recent studies have focused on the parenchymal changes surrounding DVAs. In one study, locoregional atrophy, white matter changes, cavernous malformations (CM) and dystrophic calcifications were found surrounding two thirds of DVAs (3). Another study showed that $12.5 \%$ of the lesions had subjacent fluid-attenuated inversion recovery (FLAIR) abnormalities (6).

Dehkharghani et al.(1), presented 6 cases with unilateral caudate and putamen calcifications in DVA drainage territories. All these authors believed that venous hypertension was responsible for these abnormalities. Decrease in outflow, usually caused by the stenosis of the collector vein causes chronic increase in the venous pressure within the DVA (5). Venous hypertension can also occur even without frank stenosis of the collector veins. Progressive thickening and hyalinization of the walls of DVA vessels results in increased resistance, decreased compliance and venous hypertension $(1,3)$. Chronic venous hypertension brings about chronic ischemia, edema, microhemorrhages, gliosis, dystrophic calcifications, and atrophy. Indeed, CMs have been shown to be caused by repeated microhemorrhages surrounding DVAs (4). Only 7 cases of unilateral caudate and putamen calcifications associated with DVAs that have deep venous drainage have been reported $(1,3)$. The occurrence of dystrophic calcifications in the drainage territory of DVAs drained into deep venous system rather than those drained into superficial venous system can be explained by the higher venous pressure in the former. Indeed, deep venous drainage is an increased risk factor for hemorrhage in an arteriovenous malformation related to increased intranidal pressure (8). On the contrary, in our first case, drainage was to superficial venous system. However, in our patient, a narrowing of the collector vein at the site of drainage into superficial venous system was noted, although it was not very reliably shown due to the lack of angiography or 3D T1-weigted post contrast images. We can nevertheless postulate that narrowing of the collector vein might have caused chronic venous hypertension, subsequent venous congestion, parenchymal injury and dystrophic calcification. Our first case represents the only example of unilateral putamen and caudate calcification in the vascular territory of a DVA drained into superficial ve- nous system and shows that this pattern of calcification can be seen regardless of the drainage site of the DVA. It also provides further evidence to the concept that chronic venous hypertension might be the mechanism causing parenchymal changes surrounding DVAs.

Bilateral punctate basal ganglia calcifications, usually involving globus pallidi symmetrically, is considered physiological. It has an incidence of $0.3-1.5 \%$ and is seen more frequent in elderly $(2,9)$. Metabolic diseases with abnormal calcium/phosphate balance, infection or inflammation, ischemic or toxic injury are the main reasons for bilateral calcifications in corpus striatum. Differential diagnosis of unilateral corpus striatum calcification is more limited and includes unilateral involvement by infection or neoplasm, in which case calcification is expected to be irregular (1). However, in both of our cases, basal ganglia calcifications were coarse but well- circumscribed and regular and there were no imaging or laboratory findings to suggest metabolic, infectious or neoplastic processes.

Although CT is the method of choice to diagnose calcification, it is being more frequently noticed on MRI scans especially after the routine use of GRE or SWI sequences has become common in daily practice. Most DVAs are diagnosed incidentally by routine contrast enhanced MRI scans or by SWI, even without the use of contrast agent (10). We may expect that increasingly higher number of cases with unilateral or asymmetrically dominant corpus striatum calcifications will be noticed through the routine use of GRE or SWI images. Therefore, it becomes important to know that these calcifications may be related to DVAs.

In conclusion, unilateral caudate and putamen calcifications in the drainage territory of DVAs have rarely been reported. It is postulated that chronic venous hypertension, usually caused by the outflow restriction of the collector vein, leads to parenchymal injury and dystrophic calcification. It is expected that this pattern of calcification will be more frequently recognized by MRI after the routine use of GRE or SWI sequences. Therefore, it is of great importance to be aware of this entity to avoid unnecessary diagnostic work-up and to relieve patient anxiety.

\section{Authors' Contributions}

Study concept and design: Ali Firat Sarp, Ozan Batki and Mustafa Fazil Gelal. Analysis and interpretation of data: Ali Firat Sarp and Mustafa Fazil Gelal. Drafting of the manuscript: Ali Firat Sarp and Mustafa Fazil Gelal. Critical revision of the manuscript: Mustafa Fazil Gelal. Study supervision: Mustafa Fazil Gelal. Literature search: Ali Firat Sarp and Mustafa Fazil Gelal. Guarantor: Ali Firat Sarp.

\section{References}

1. Dehkharghani S, Dillon WP, Bryant SO, Fischbein NJ. Unilateral calcification of the caudate and putamen: association with un- 
derlying developmental venous anomaly. AJNR Am J Neuroradiol. 2010;31(10):1848-52.

2. Osborn AG, Blazer S, Salzman KL. Diagnostic imaging: brain. 1 edSalt Lake City: Amirsys; 2004.

3. San Millan Ruiz D, Delavelle J, Yilmaz H, Gailloud P, Piovan E, Bertramello A, et al. Parenchymal abnormalities associated with developmental venous anomalies. Neuroradiology. 2007;49(12):987-95.

4. Dillon WP. Cryptic vascular malformations: controversies in terminology, diagnosis, pathophysiology, and treatment. AJNR Am J Neuroradiol.1997;18(10):1839-46.

5. Pereira VM, Geibprasert S, Krings T, Aurboonyawat T, Ozanne A, Toulgoat F, et al. Pathomechanisms of symptomatic developmental venous anomalies. Stroke. 2008;39(12):3201-15.

6. Santucci GM, Leach JL, Ying J, Leach SD, Tomsick TA. Brain parenchymal signal abnormalities associated with developmental venous anomalies: detailed MR imaging assessment. AJNR Am J Neuroradiol. 2008;29(7):1317-23.

7. Forsting M, Wanke I. Developmental Venous Anomalies. In: Forsting $\mathrm{M}$ editor. Intracranial vascular malformations and aneurysms : from diagnostic work-up to endovascular therapy,. 1 ed. Berlin: Springer; 2006. pp. 1-13.

8. Duong DH, Young WL, Vang MC, Sciacca RR, Mast H, Koennecke $\mathrm{HC}$, et al. Feeding artery pressure and venous drainage pattern are primary determinants of hemorrhage from cerebral arteriovenous malformations. Stroke.1998;29(6):1167-76.

9. Kiroglu Y, Calli C, Karabulut N, Oncel C. Intracranial calcifications on CT. Diagn Interv Radiol. 2010;16(4):263-9.

10. Mittal S, Wu Z, Neelavalli J, Haacke EM. Susceptibility-weighted imaging: technical aspects and clinical applications, part 2. AJNR Am J Neuroradiol. 2009;30(2):232-52. 